\title{
T Wave Alternans in high arrhythmic risk patients: Analysis in time and frequency domains: A pilot study Anthony C Hunt
}

Address: Medical Director, Cardio-Analytics, ITTC 2, Tamar Science Park, Davy Road, Derriford, Plymouth, UK PL6 8BX

E-mail: tony.hunt@talk21.com

Published: 12 March 2002

BMC Cardiovascular Disorders 2002, 2:6

This article is available from: http://www.biomedcentral.com/|47|-226|/2/6

(C) 2002 Hunt; licensee BioMed Central Ltd. Verbatim copying and redistribution of this article are permitted in any medium for any purpose, provided this notice is preserved along with the article's original URL.

\begin{abstract}
Background: $T$ wave alternans (TA) is a repolarisation phenomenon manifesting as a microvolt beat to beat change in the amplitude of the T wave and ST segment. TA has been shown to be a predictor of arrhythmic risk in unselected myocardial infarction populations. TA has not been used to differentiate risk within the ischaemic cardiomyopathy population.
\end{abstract}

Methods: The subjects investigated comprised, Group I: 7 stable patients with remote $(>20$ months) extensive myocardial scarring and no arrhythmic events (NYHA 3 and 4). Group2: 9 post infarction patients with malignant arrhythmia and implantable defibrillator. During breath holding, 20 continuous $Q R S T$ complexes from each patients $X, Y$ and $Z$ leads were digitally recorded. Time domain, resultant absolute difference vectors (ATA), were calculated for alternate resultant $T$ wave sequences. Group differences between the magnitude and temporal distribution of mean ATAs and their spectral and cross-spectral analysis were compared.

Results: Group I v Group 2 mean ATAs were 10.7 (7.17) v II.7 (8.48) respectively, not significant. Each group had a homogenous temporal distribution of ATAs. Both group's largest mean ATA frequency components were between 0 to $25 \mathrm{~Hz}$, the largest ATA component being at the DC frequency. Cross spectral analysis showed no significant differences in group ATA frequency content.

Conclusion: The frequency content and microvolt magnitude of $T$ wave alternans was not significantly different in these two groups. The specificity of $T$ wave alternans for differentiating arrhythmic risk in post infarction scarring and heart failure needs investigation.

\section{Background}

$\mathrm{T}$ wave alternans (TA) is a repolarisation phenomenon manifesting as a microvolt beat to beat change in amplitude of the T wave and ST segment. The mechanism responsible for TA is a dynamic (beat to beat) variation of the repolarisation sequence [1] due to either an alternation of action potential duration, frequently associated with the long QT syndrome [2] or an alternation of the time course of repolarisation [3] frequently associated with ischaemia [4]. The alternation of time course and multilevel distribution of electrical repolarisation activity during ischaemia [4] cause the electrocardiographic manifestation of TA in addition to alternating dispersion through islands of partially and fully refractory tissue which promote wavefront fractionation and vulnerability to reentrant malignant arrhythmias (MA) $[5,6]$. 
The earliest description of TA was made in 1910 by Lewis [7]. Animal work by Hellerstein and Liebow showed a mechanistic link between TA and arrhythmogenesis [8] and recently the presence of TA has been recognised as being a harbinger of sudden death $[9,10]$. Rosenbaum et al reported TA to be an independent prognosticator of vulnerability to arrhythmias predicted by electrophysiology testing[11] and these findings were repeated by Armoundas and co-workers showing TA to be superior to signal average electrocardiography as a predictor of malignant ventricular arrhythmias in high risk populations [12]. TA has demonstrated high sensitivities and specificities as a predictor of arrhythmic risk in unselected myocardial infarction populations [13] and idiopathic cardiomyopathy [14] but TA has not been used to differentiate risk in the ischaemic cardiomyopathy group.

The purpose of this pilot study was to use indices of absolute micro voltage TA and the frequency content of alternating $\mathrm{T}$ waves to assess the ability to differentiate post infarction heart failure patients with significant substrates for developing re-entrant MA but without arrhythmic events, from those post infarction patients at most risk from developing MA.

\section{Materials and Methods}

Approval was obtained from the local Ethics Committee prior to performing the described research. The research was conducted in accordance the Helsinki Declaration, written informed consent was obtained from each patient prior to collecting data. The study population consisted of two groups of stable treated patients: Group 1 comprised 7 patients with extensive scarring secondary to myocardial infarction and compensated treated left sided heart failure with mean ejection fraction of 35\%. The patient with the most recent infarct event in this group occurred over 20 months prior to the study. No patient within group 1 sustained a symptomatic arrhythmic event. Group 2 comprised 9 patients with a history of myocardial infarction with a mean ejection fraction of $38 \%$, a distant episode of sustained ventricular tachycardia or aborted sudden death, positive induction of sustained ventricular tachycardia during an electrical stimulation study and a history of prophylactic implantation of an automatic defibrillator.

20 sequential electrocardiographic complexes were recorded from $\mathrm{X}, \mathrm{Y}$ and $\mathrm{Z}$ orthogonal leads on each resting supine patient during a held half expiration breath without straining. The ECG recordings were made using and analog-to-digital converter (Powerlab), sampling at 1000 $\mathrm{Hz}$. All the recorded signals were not filtered, because it was intended to make inter-group statistical comparisons in the frequency domain between the higher frequency band of 80-500 Hertz, where there may exist representations of possible late potential repolarisation. As all signif- icant non-noisy signals have been found to occur within a 0 to 40 Hertz pass-band [15], the recorded signals underwent 40 Hertz low pass filtering prior to analysis in the time domain.

For each lead on each patient, sequences of the alternate ST-T waves were ensemble averaged over a 400 millisecond duration using the positive or negative peak of the $\mathrm{T}$ wave as the fiducial central point of data collection over 400 milliseconds, see figure 1 . In leads where the $\mathrm{T}$ wave was of low magnitude, the fiducial central point was taken to occur as the point at an average of the times from the peak QRS deflection to the fiducial T wave point in the other clearly demarcated orthogonal leads. The vectors of the squared magnitude of differences in ensemble averaged time aligned alternating ST-T sequences were calculated on each lead from each patient. For each patient, the resultant absolute difference vector (ATA) was calculated as the square root of the summed squared differences between alternate ensemble averaged $T$ waves for each $\mathrm{X}, \mathrm{Y}$ and $Z$ vector. All calculations were performed using Mathcad Professional signal processing software on a Acer Pentium 2 PC. Comparisons were made between each mean group ATA as well as between the intra and inter-group means of vectors representing each third time interval of the respective mean ATAs. The spectral content was calculated for each group mean ATA by digital fast Fourier algorithm and cross spectral analysis was calculated between each group mean ATA to assess differences in the frequency components within each group. In order to assess group differences in the 80 to 500 Hertz frequency band, mean magnitude and standard deviation of the 80 to 500 $\mathrm{Hz}$ frequency band in the group with the smallest mean magnitude was considered noise and group differences between mean magnitudes in this band only considered significant if they exceeded three times the previously calculated standard deviation.

The following describes the method by which the digitised ensemble averaged alternate $T$ wave vectors $(X 1, X 2$, $\mathrm{Y} 1, \mathrm{Y} 2, \mathrm{Z} 1$ and $\mathrm{Z} 2$ ) were used to calculate the mean ATAs for each group.

Time of $\mathrm{T}$ wave Analysis $=\mathrm{t}=400$ milliseconds

Subjects in groups 1 and $2=1$ to $\mathrm{N} 1$ and 1 to N2

$[\text { Vector X1 - Vector X2 }]^{2}=$ Vector $(\text { DiffX })^{2}$

[Vector Y1 - Vector Y2 $]^{2}=$ Vector $(\text { DiffY })^{2}$

[Vector Z1 - Vector Z2 $]^{2}=$ Vector $(\text { DiffZ })^{2}$

Resultant Absolute Difference Vector $($ ATA $)=\sqrt{ }\left(\right.$ DiffX $^{2}+$ DiffY $^{2}+$ DiffZ2) $^{2}$ 
Group1 mean ATA $=\sum_{1}^{\mathrm{N} 1}(\mathrm{ATA}) /(\mathrm{N} 1) \quad$ Group2 mean ATA $=\sum_{1}^{\mathrm{N} 2}(\mathrm{ATA}) /(\mathrm{N} 2)$

Mean value of Group (1 or 2) mean ATA= [Group (1 or 2) mean ATA]/t

\section{Statistics}

Two tailed t test was used to assess group differences between mean ATAs and intra and inter-group differences between the first (0-133 milliseconds), second (134-237 milliseconds) and third mean ATA (238-400 milliseconds). The statistical group differences between the magnitude of components within the 80 to $500 \mathrm{~Hz}$ band have been described above.

\section{Results}

An example of the time aligned alternate sequences of STT waves over 400 milliseconds duration is shown in figure 2. The mean value and standard deviation for each group member ATA are shown in Table 1 . There was no significant difference between the means for groups 1 and 2 mean ATAs. The mean value and standard deviation for group $1 v$ group 2 being 10.7 microvolts (standard deviation $=7.17$ microvolts) $v 11.74$ microvolts (standard deviation= 8.48 microvolts) respectively. All the values for the difference between ensemble averaged $\mathrm{T}$ waves for each vector in each patient are shown in table 1 . There was no intra or inter-group significant difference in the mean values between the first, second or third time intervals for each groups mean ATA. The mean values for first, second and third intervals for Group $1 v$ Group 2 being $6.59 \mathrm{v}$ $7.17,6.37 v 7.96$ and 6.06 v 6.18, respectively. The Power Spectrum for each groups resultant absolute difference vector between alternate sequences of ensemble averaged ST-T waves showed the largest values between 0 and 25 Hertz with maximum significant differences at the mean or DC frequency, see figures 3 and 4 . The cross spectral density function showed that both groups shared the same frequency components, see figure 5. There was no significant group differences between the mean magnitudes of frequency band 80-500 Hertz for Group $1 v$ Group 2. The standard deviation in this band for Group 1 (noise) $=0.562$, the mean magnitudes for Group $1 \mathrm{v}$ Group 2 in this band being 0.615 v 0.823 .

\section{Discussion}

The purpose of this pilot study was to assess the ability of the absolute measurements for the microvolt magnitude of TA to differentiate between one group with an established high risk of MA (Group2) versus a group of patients with multiple physical substrates for developing MA but without a history of a significant arrhythmic event (Group 1 ). Group 1 was chosen to be representative of those pa-
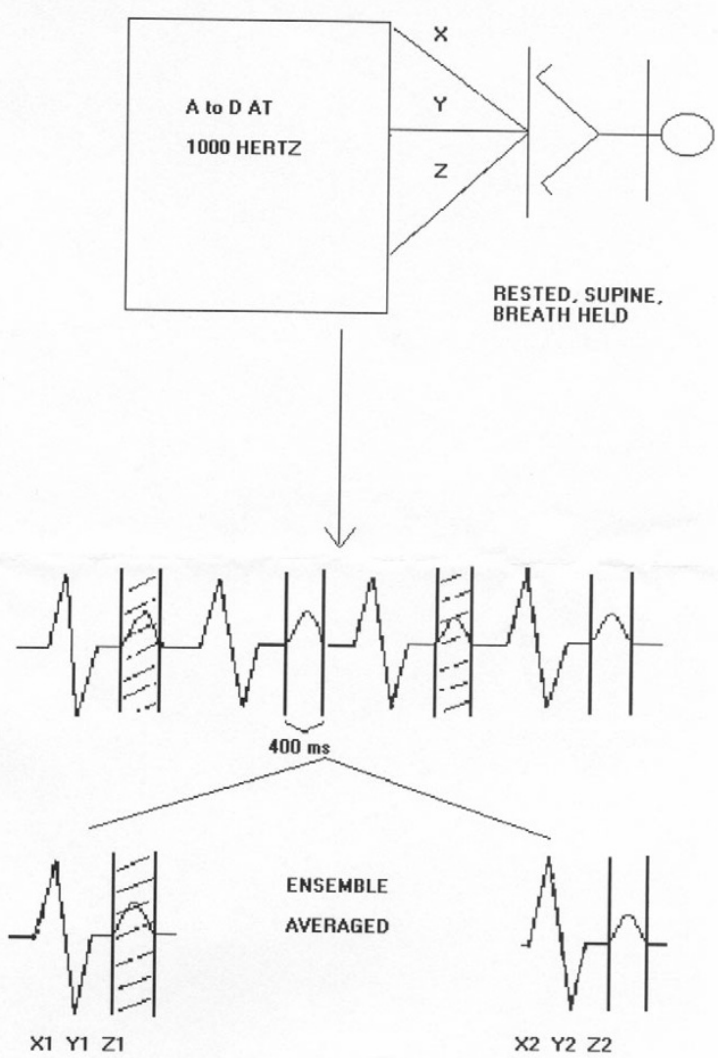

Figure I

Diagram showing methodology for sampling analog ECG signal in $X, Y$ and $Z$ leads in a rested, supine patient during breath holding. 400 milliseconds of alternate $T$ wave sequences were ensemble averaged for each lead and collected in matrix bins represented by $X I, Y I, Z I$ and $X 2, Y 2$ and $\mathrm{Z} 2$.

tients whom make up a large proportion of the inexorably increasing coronary heart disease population and on clinical criteria would theoretically be at highest risk from developing a MA and therefore most likely to benefit from accurate risk stratification and defibrillator implantation. It is important to scrutinise the limitations of any index proffered to help risk stratification and aid the focussing of limited resources of invasive electrophysiological investigation with a view to device therapy.

Assuming a standard error of approximately 6\% for the measurement of ejection fraction by acoustic quantification method, the two groups had similar ejections fractions and presumably similar degrees of post-infarction ventricular damage. The lower ejection fraction of Group 1 would be compatible with the intended selection bias 


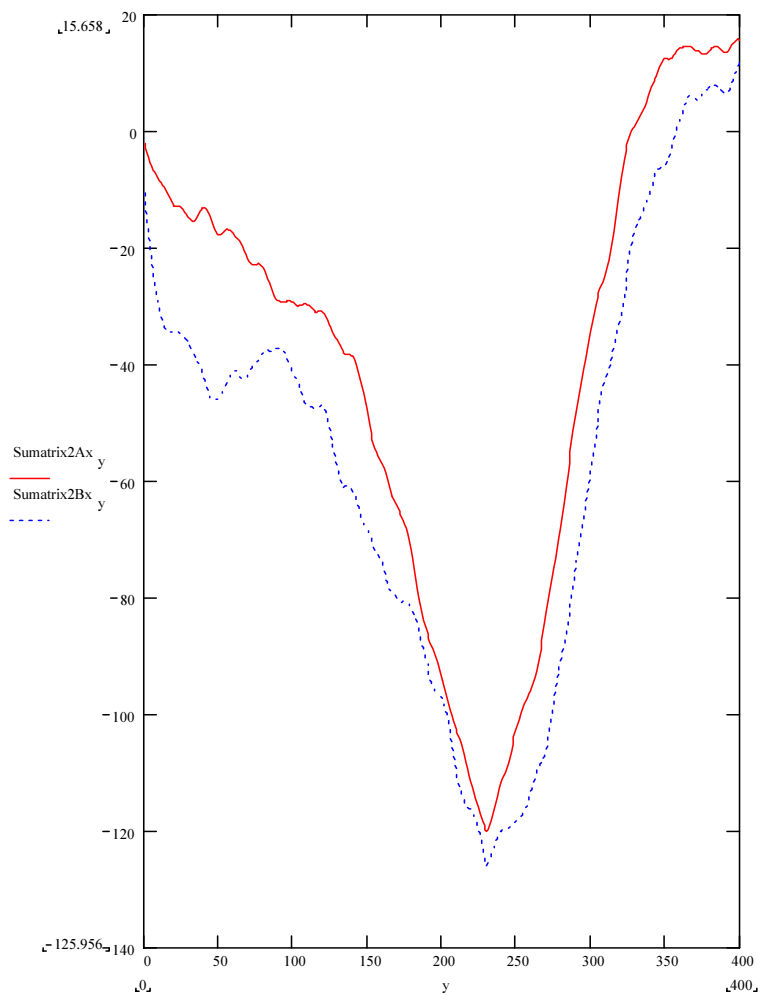

\section{Figure 2}

An example of two ensemble averaged, time aligned, alternate 400 milliseconds of $T$ wave sequences for lead $X$ in subject 2. The $x$ axis is in milliseconds and the $y$ axis in microvolts. The dashed and continuous tracings represent an inverted $\mathrm{T}$ wave in this subject.

and the greater degree of scarring and contractile dysfunction function in this group. Both groups were stable in terms of heart failure and the absence of dynamic symptomatic ischaemic syndromes, therefore the observed measurements of TA in time and frequency domains are expected to arise from mechanism associated with the post myocardial infarction scarred tissue and it's immediate periphery.

Conventional TA indices either measure the alternans ratio [11] which is the extent to which the measured alternans exceeds the uncertainty (noise) of the measurement and conveys the statistical degree of confidence in the alternans measurement, or the cumulative alternans voltage in microvolts, which is derived from the frequency power spectrum and analogous, but not equivalent to resultant vector of absolute difference in alternate $T$ wave sequences (ATA) used as the index in this study. The spectral method is an indirect method which measures the magnitude of TA as the amplitude of spectral power at the 0.5 cycles per beat frequency and then subtracts the mean background noise.

This method will not compensate for the presence of superposed noise contamination of the 0.5 frequency component amplitude which is then squared to derive the power term. There is an assumption with the spectral measurement method, that the noise is all broad band but it may be non-linear or multiplicative and therefore not eliminated by the spectral method of subtracting the mean of the baseline noise. Perturbations to increase the heart rate have been used to improve the signal to noise ratio of the spectral method in order to improve the sensitivity to detect significant TA. This study describes a direct method which measures absolute TA which for reasons described in the next paragraph has a high signal to noise ratio. This may account for the higher mean values of ATA compared to the values of mean cumulative alternans voltage which is circa 1.2 microvolts. As the index ATA is an absolute measure of the alternating microvolt T wave amplitude, what it conveys is more in faith with the

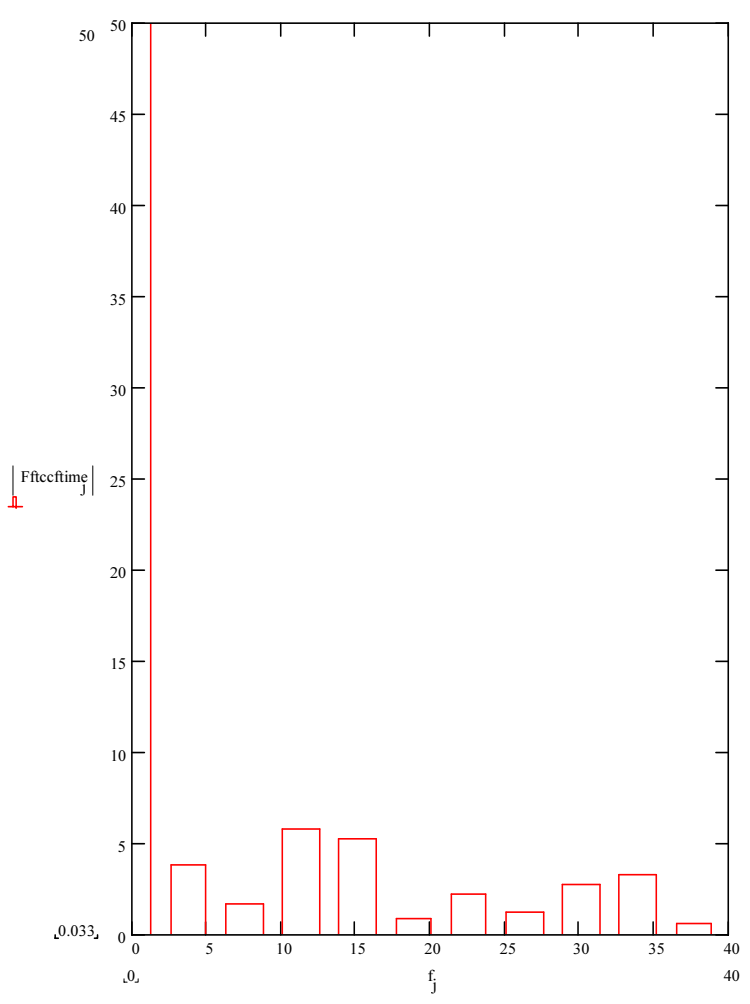

Figure 3

Spectral analysis of the resultant vectored absolute mean TA for the ischaemic cardiomyopathy group (group I). The $y$ axis represents the magnitude of spectral components and the $x$ axis represents frequency in Hertz. Only the range from 0 to 40 Hertz is shown for clarity. 
definition of TA. This pilot study demonstrates that the resultant vector of absolute differences in TA microvoltage measured as the index ATA, which was obtained from ensemble averaged alternate $T$ wave sequences during breath holding, may demonstrate low specificity when applied to the risk stratification in those post-infarction patients with ischaemic cardiomyopathies. A recent study by Sakabe and colleagues have demonstrated a specificity of only $35 \%$ in a prospective study using TA to predict the development of ventricular tachycardia in non-ischaemic cardiomyopathy patients [16]. This paper supports our results and has significant implications for the specificity of TA when used as a discriminatory probe in the cardiomyopathy patient subset.

Our method of measuring absolute TA minimises inaccuracies due to noise by ensemble averaging the data collected under optimal conditions of stability of the electrocardiographic tracing by suspending respiration in a supine rested patient. The noise remaining after ensemble averaging is then largely eliminated by subtracting the

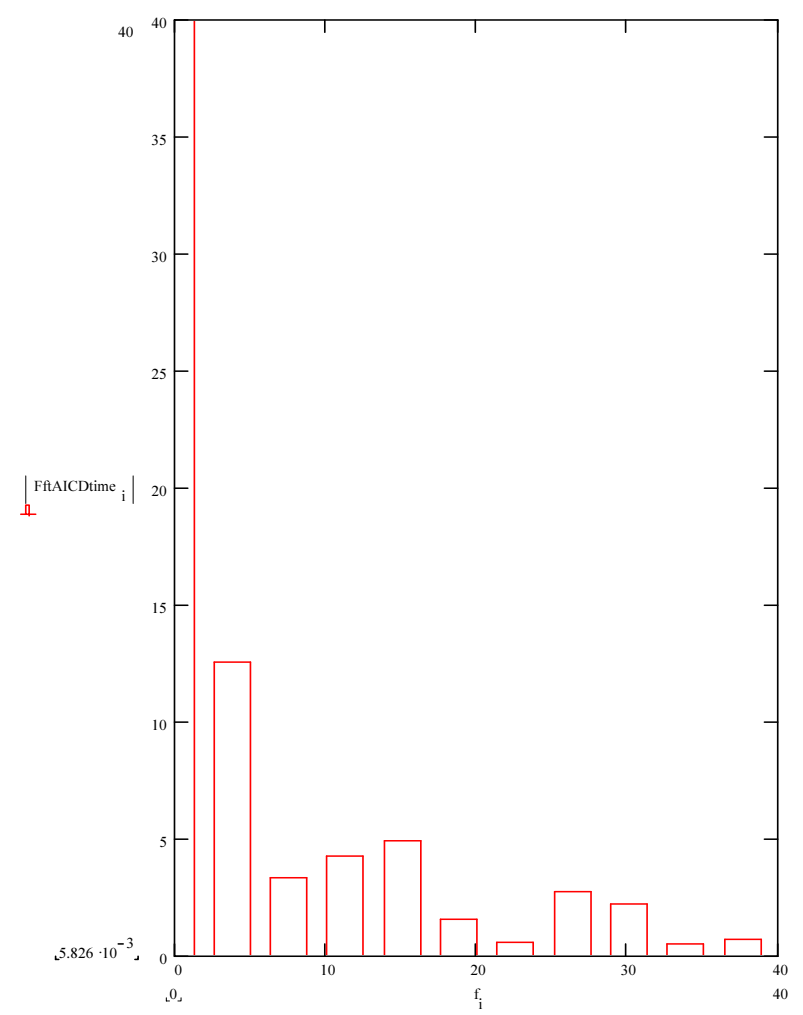

\section{Figure 4}

Spectral analysis of the resultant vectored absolute mean TA for the implanted defibrillator group (group 2). The $y$ axis represents the magnitude of spectral components and the $x$ axis represents frequency in Hertz. Only the range from 0 to 40 Hertz is shown for clarity.

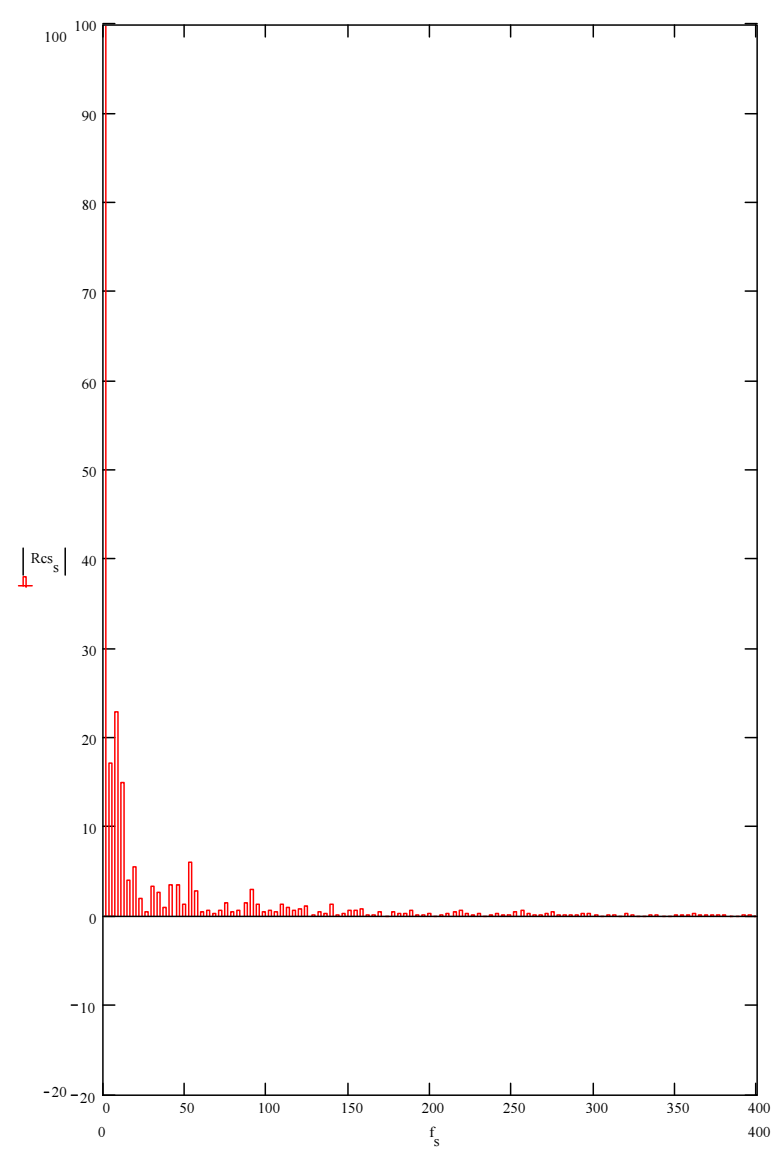

Figure 5

Cross-Spectral analysis for the resultant vectored absolute mean TA for both groups. The $y$ axis is the magnitude of cross-spectral frequency components and the $x$ axis is in Hertz. Note that all positive values indicate the presence of a common frequency component between both groups with expected large magnitudes in the frequency range between 0 to 40 Hertz.

alternate ensemble averaged $\mathrm{T}$ wave signal sequences from each other, thus subtracting the equal amounts of background noise within each of the sequences from each other. It is known that TA can be produced by motion of the heart within the thoracic cavity, previous studies have not addressed the augmentation of such motion with respiration. It has been suggested that using a resultant vector of orthogonal leads is sufficient to mitigate against the effects of mechanical rotation of the heart when measuring TA [11] but theoretically the resultant vector will oscillate with cardiac and respiratory motion. Data from long, noisy, non-stationary ECG recordings, of the type found in Holter recordings or during exercise, would add to inaccuracies resulting from cardiac motion. We attempted to correct for any such inaccuracy arising from respiration- 
Table I: Values of the squared microvolt differences between ensemble averaged $T$ waves meaned over time, in each patients $X$, $Y$ and $Z$ leads and values of each patients ATA. Patient numbers $I$ to 7 represent Group I and patient numbers 8 to 16 represent Group 2. Values $<0.015$ rounded to 0 .

\begin{tabular}{lllll}
\hline & $X$ & $Y$ & $Z$ & ATA \\
\hline 1 & 304.85 & 0 & 72.76 & 19.43 \\
2 & 0 & 0 & 0 & 0 \\
3 & 144.96 & 0 & 94.67 & 15.48 \\
4 & 24.6 & 0 & 0 & 4.96 \\
5 & 38.8 & 55.65 & 0 & 9.72 \\
6 & 4.6 & 44.62 & 7.07 & 7.5 \\
7 & 192.93 & 32.26 & 91.58 & 17.8 \\
8 & 143 & 278.9 & 0 & 20.05 \\
9 & 2.16 & 2.16 & 17.5 & 4.67 \\
10 & 40.57 & 0 & 6.66 & 6.87 \\
11 & 186.6 & 0 & 0 & 13.66 \\
12 & 57.5 & 31.92 & 33.9 & 11.1 \\
13 & 0 & 0 & 0 & 0 \\
14 & 0 & 17.3 & 0 & 4.16 \\
15 & 225.6 & 291.7 & 9.36 & 22.9 \\
16 & 19.45 & 320.41 & 133.9 & 21.8 \\
& & & & \\
\hline
\end{tabular}

by suspending the respiration in half held breath. The number of recordings of complexes was limited due to the time over which the patients could comfortably breathhold but the accuracy of the measured absolute resultant vector of microvolt TA in a limited number of complexes was considered an advantage over a TA index compiled from a more numerous but more variable and inaccurate data source.

For both groups the absolute difference in mean ATA was distributed equally in time throughout the ST-T wave, which corresponds to the finding that the maximum differences in frequency content for the ATAs in both groups occur at the mean or dc frequency component but there was also a significantly increased magnitude of ATA Within the 0-25 Hertz frequency band which would include the $T$ wave peak frequency and subharmonics within the T wave. In the same way that spatial heterogeneities of depolarisation are responsible for the spectral component markers of late potentials within the QRS complexes, Fourier techniques were applied to the ST-T waves to determine the presence of any difference in the higher frequency bands between alternating ST-T sequences which may indicate the presence of alternating spatial heterogeneities of repolarisation. The finding of no statistically significant group differences in mean magnitude within the frequency band 80 to $500 \mathrm{Hertz}$ is compatible with the findings of Steinbigler et al [15] and does not provide a probe to differentiate these two groups. This study only showed a significant difference in the mean fre- quency of alternating sequences, which suggests that the differences in measured alternating microvolt magnitudes of the ST-T wave were not due to a change in the morphology of the wave due to introduction of discrete frequency components. The ATAs in the time domain also showed homogenous distribution throughout the ST-T waves, which implies that the origin of TA is not an anatomically localised phenomena as it does not appear to arise from the repolarisation gradient generated between the epicardial, endocardial and M myocardial layers, which according to Antzelevitch and colleagues correspond to the epochs of the start of the peak T wave, the initial descending $\mathrm{T}$ wave and end of the descending $\mathrm{T}$ wave respectively [17].

There is controversy as to whether TA indices show significant specificity when identifying patients with idiopathic cardiomyopathy at high risk of ventricular arrhythmias $[14,16]$. Alternatively, TA has been shown to be very useful in identifying arrhythmic risk in the non-specific post infarction population, where sensitivities and negative predictive values are $93 \%$ and $98 \%$ respectively [13]. However, the major arrhythmic risk lies in the large post infarction cardiomyopathy population but there has been little focus on the use of TA markers in this specific subgroup with coronary heart disease. On the basis of this pilot study, larger scale studies are indicated with electrophysiological ventricular stimulation as reference standard to further assess the sensitivity and specificity of absolute and spectral derived indices of TA to identify patients at high risk of MA within the population of post infarction ischaemic cardiomyopathic heart failure patients.

\section{Conclusions}

This pilot study did not show any significant difference in the magnitude and distribution of absolute TA microvoltages or spectral components between Groups 1 and 2 . Our results in ischaemic cardiomyopathy patients is supported by another study on cardiomyopathy subsets which has shown a low specificity for TA to identify arrhythmic risk. These results suggest larger scale studies are needed.

\author{
Abbreviations \\ $\mathrm{TA}=\mathrm{T}$ wave alternans \\ ATA $=$ Resultant absolute difference vectors \\ $\mathrm{MA}=$ malignant arrhythmia
}

\section{Competing interests}

None declared. 


\section{References}

I. De Ambroggi L: Heterogeneities of ventricular repolarisation and vulnerability to arrhythmia. How to detect them with non-invasive methods? Cardiologica 1999, 44(4):355-60

2. Surawicz B, Fisch C: Cardiac Alternans: diverse mechanisms and clinical manifestations J Am Coll Cardiol 1992, 20(2):483-99

3. Pastore JM, Girouard SD, Laurita KR, et al: Mechanism linking $\mathbf{T}$ wave alternans to the genesis of cardiac fibrillation Circulation 1999, 99(10): 1 385-94

4. Green LS, Fuller MP, Lux RL: Three dimensional distribution of ST-T wave alternans during acute ischaemia / Cardiovasc Electrophysiology 1997, 8(1 2): |413-9

5. Smith JM, Cohen RJ: Simple finite-element model accounts for wide range of cardiac dysrhythmias Proc Nat Ac ad Sci Med Sci 1984, 8 I:233-7

6. Clancy EA, Smith JM, Cohen RJ: A simple electrical mechanical model of the heart applied to electrical-mechanical alternans IEEE Trans Biomed Eng 1991, 38:551-60

7. Lewis T: Notes upon alternation of the heart $Q J$ Med 1910, 4:|4|-4

8. Hellerstein HK, Liebow IM: Electrical alternation in experimental coronary artery occlusion Am J Physiol 1950, 160:366-74

9. Schwartz PJ, Milliani A: Electrical Alternation of the $\mathbf{T}$ wave: clinical and experimental evidence of its relationship with the sympathetic nervous system and with long QT syndrome Am Heart J 1975, 89:45-50

10. Raeder EA, Rosenbaum DS, Bhasin R, et al: Alternating morphology of the QRST complex preceding sudden death $N$ Engl J Med 1992, 326:271-2

I I. Rosenbaum DS, Jackson LE, Smith JM, et al: Electrical Alternans and vulnerability to ventricular arrhythmias $N$ Engl J Med I 994, 330(4):235-4I

12. Armoundas AA, Rosenbaum DS, Ruskin JN, et al: Prognostic significance of electrical alternans versus signal averaged electrocardiography in predicting the outcome of electrophysiological testing and arrhythmia free survival Heart 1998, 80:251-56

13. Ikeda T, Sakata T, Takami M, et al: Combined assessment of Twave alternans and late potentials used to predict arrhythmic events after myocardial infarction. A prospective study J Am Coll Cardiol 2000, 35(3):722-30

14. Adachi K, Ohnishi Y, Shima T, et al: Determinant of microvoltlevel $\mathbf{T}$ wave alternans in patients with dilated cardiomyopathy J Am Coll Cardiol 1999, 34(2):374-80

15. Steinbigler $P$, Haberl $R$, Nespithal $K$, et al: T wave spectral variance, a new method to determine inhomogeneous repolarisation by $T$ wave beat-to-beat variability in patients prone to ventricular arrhytrhmias / Electrocardiology 1998, 30: |37-|44

16. Sakabe K, Ikeda T, Sakata T, et al: Comparison of T wave alternans and $Q T$ interval dispersion to predict ventricular tachyarrhythmia in patients with dilated cardiomyopathy Jpn Heart J 200I, 42(4):45I-7

17. Antzelevitch C, Shimizu W, Yan GX, et al: Cellular basis for QT dispersion / Electrocardiology. 1998, 30(Suppl): |68-75
Publish with BioMed Central and every scientist can read your work free of charge

"BioMedcentral will be the most significant development for disseminating the results of biomedical research in our lifetime."

Paul Nurse, Director-General, Imperial Cancer Research Fund

Publish with BMC and your research papers will be:

- available free of charge to the entire biomedical community

- peer reviewed and published immediately upon acceptance

- cited in PubMed and archived on PubMed Central

- yours - you keep the copyright

Submit your manuscript here:

http://www.biomedcentral.com/manuscript/
BioMedcentral.com editorial@biomedcentral.com 